\title{
Comparing Three Data Mining Algorithms for Identifying the Associated Risk Factors of Type 2 Diabetes
}

\author{
Habibollah Esmaeily ${ }^{1}$, Maryam Tayefi ${ }^{2}$, Majid \\ Ghayour-Mobarhan ${ }^{3}$ and Alireza Amirabadizadeh ${ }^{4 *}$ \\ ${ }^{1}$ Department of Biostatistics, School of Health, Mashhad University of Medical Sciences, Mashhad, Iran; \\ ${ }^{2}$ Department of Modern Sciences and Technologies, School of Medicine, Mashhad University of \\ Medical Sciences, Mashhad, Iran; ${ }^{3}$ Biochemistry of Nutrition Research Center, School of Medicine, \\ Mashhad University of Medical Sciences, Mashhad, Iran; ${ }^{4}$ Medical Toxicology and Drug Abuse Research Center \\ (MTDRC), Birjand University of Medical Sciences, South Khorasan, Iran
}

Received 24 September 2017; revised 26 November 2017; accepted 9 December 2017

\begin{abstract}
Background: Increasing the prevalence of type 2 diabetes has given rise to a global health burden and a concern among health service providers and health administrators. The current study aimed at developing and comparing some statistical models to identify the risk factors associated with type 2 diabetes. In this light, artificial neural network (ANN), support vector machines (SVMs), and multiple logistic regression (MLR) models were applied, using demographic, anthropometric, and biochemical characteristics, on a sample of 9528 individuals from Mashhad City in Iran. Methods: This study has randomly selected 6654 (70\%) cases for training and reserved the remaining 2874 (30\%) cases for testing. The three methods were compared with the help of ROC curve. Results: The prevalence rate of type 2 diabetes was $14 \%$ in our population. The ANN model had $78.7 \%$ accuracy, $63.1 \%$ sensitivity, and $81.2 \%$ specificity. Also, the values of these three parameters were $76.8 \%, 64.5 \%$, and $78.9 \%$, for SVM and $77.7 \%, 60.1 \%$, and $80.5 \%$ for MLR. The area under the ROC curve was 0.71 for ANN, 0.73 for SVM, and 0.70 for MLR. Conclusion: Our findings showed that ANN performs better than the two models (SVM and MLR) and can be used effectively to identify the associated risk factors of type 2 diabetes. DOI: 10.29252/ibj.22.5.303
\end{abstract}

Keywords: Data mining, Diabetes type 2, Support vector machine,

Corresponding Author: Alireza Amirabadizadeh

Medical Toxicology and Drug Abuse Research Center (MTDRC), School of Medicine, Birjand University of Medical Sciences, Birjand, Iran;

Tel.: (+98-910) 5090341; Fax: (+98-563) 2381250; E-mail: amirabadiza921@gmail.com

\section{INTRODUCTION}

$\mathrm{D}$ iabetes mellitus (DM) is rapidly growing in prevalence, thus posing a major public health challenge globally. The estimated number of diabetic adults was 382 million in 2013, while the prediction for year 2035 is 592 million $^{[1,2]}$. Diabetes is associated with health complications such as cardiovascular, renal, and retinal diseases; therefore, it is important to identify individuals having a high risk of diabetes, in order to control other health risks.

Different models have been developed during the last two decades for evaluating the risk of DM, but none of them have been found to achieve the desired accuracy, hence having a limited clinical utility ${ }^{[3,4]}$. Studies have also suggested an connection between anthropometric factors and $\mathrm{DM}^{[5,6]}$.

Data mining involves choosing, analyzing, and modeling huge amounts of information, aiming to reveal undisclosed patterns or correlations that propose obvious and beneficial results ${ }^{[7,8]}$. This method has progressed fast in the recent years. Several researches have used data mining in order to investigate unknown variables. In addition, some predictive models are created in medicine ${ }^{[9,10]}$.

The multiple logistic regression (MLP) is a nonlinear 
regression approach to predict a categorical and a dependent variable. This method has been used for identifying the risk factors for various diseases through patients' history, characteristics, and other risk factors. The logistic model measures the probability of the considered illness $\mathrm{y}(\mathrm{y}=0$ if the participant does not suffer from the illness; if not, $y=1$ ) as a function for the predictive risk factor values. If the person suffers from this illness, the conditional probability can be obtained by $\mathrm{p}(\mathrm{y}=1 \mid \mathrm{X})=\mathrm{p}(\mathrm{X})$, and the logistic model becomes: $\log [\mathrm{p}(\mathrm{x}) / 1=\mathrm{p}(\mathrm{x})]=\mathrm{B}_{0}+\mathrm{B}_{1} \mathrm{x}_{1}+$ $\mathrm{B}_{2} \mathrm{x}_{2}+\mathrm{B}_{\mathrm{k}} \mathrm{x}_{\mathrm{k}}$ in which $\mathrm{X}=\left(\mathrm{x}_{1}, \mathrm{x}_{2}, \ldots, \mathrm{x}_{\mathrm{k}}\right)$ indicates the $\mathrm{k}$ 's risk factors vector through the logistic regression model ${ }^{[11,12]}$.

The support vector machines (SVMs) create appropriate boundaries between the information sets through solving a quadratic optimization problem. By making the use of various kernel functions, different amounts of flexibility and nonlinearity can be attached to the model. Since these features could be elicited from high level statistical ideas, and their generalization error bounds can be measured, a great deal of research has been conducted on SVMs during the last years ${ }^{[13,14]}$.

The artificial neural networks (ANNs) are suitable data mining instruments that are utilized for building nonlinear and complex models. The present study utilized a common ANN architecture named multilayer perception network with back-propagation algorithm, which is surely the most widely used and carefully deliberated ANN architecture ${ }^{[15,16]}$. The multi-layer perception networks are feed-forward neural networks that are instructed with standard backpropagation algorithms and are considered as a strong function approximate for the problems of classifying and predicting ${ }^{[17]}$.

In public health, it is common to use MLR for identifying factors associated with the disease and for developing predictive models ${ }^{[18,19]}$. Some data mining techniques have already been applied for medical conditions $^{[9,20,21]}$ and for predicting diabetes ${ }^{[8,22,23]}$. The present study aims at comparing ANN, SVM, and MLR for identifying associated risk factors of DM.

\section{MATERIALS AND METHODS}

\section{Experimental sample}

A sample of 9528 subjects were enrolled in the Mashhad Stroke and Heart Atherosclerotic Disorders Study at Mashhad University of Medical Sciences (MUMS), Mashhad, Iran ${ }^{[24]}$. The Ethics Committee of MUMS has approved the protocol, and all the participants have been given an informed written consent.

\section{Data collection (anthropometric and biochemical measurements)}

Demographic characteristics consisted of age, gender, marital status, education, cigarette smoking habit, physical activity level (PAL), family history of diabetes, and depression score. The depression score was evaluated using Beck's depression inventory II. Anthropometric information included weight, height, waist, and hip circumference. Systolic and diastolic blood pressures were measured as described earlier ${ }^{[12]}$. Biochemical measurements were composed of fasting blood glucose, fasting serum triglycerides (TGs), total cholesterol (TC), HDL- and LDL-cholesterol, and high-sensitivity C-reactive protein (hs-CRP), as previously described ${ }^{[24]}$.

\section{Phenotypic definition of type 2 diabetes mellitus}

Phenotypic definition of Type 2 DM was specified based on the fasting blood glucose level of $126 \mathrm{mg} / \mathrm{dl}$ or higher.

\section{Artificial neural network}

ANN is a data mining tool and is used for constructing non-linear prediction models ${ }^{[7]}$. A multilayer neural network has three layers, namely an input layer, a hidden layer, and an output layer. Each layer has nodes that are connected by links from one layer to the next. Nodes in the input layer represent predictors, while in the output layer, the nodes are viewed as outcome variables ${ }^{[15]}$. One of the most applications of neural network is multilayer backpropagation learning algorithm that has ability of modeling a non-linear systems ${ }^{[25]}$. Interpretation of neural networks is more complicated than other statistical models; however, the neural network is used in different medical fields ${ }^{[25,26]}$. The structure of perceptron network is composed of some nodes with an activation function that are held in different layers. Each node by its weight coefficients collects the results of all previous nodes and converts it to next layer through activation function. The number of nodes in each layer of network depends on the structure of the investigated subject ${ }^{[15,25]}$. In a perceptron network with a hidden layer, the amount of $i$-th output is calculated using the following formula in which $\mathrm{n}$ : the number of observations, $\mathrm{M}$ : the number of hidden layer nodes, $\mathrm{p}$ : the number of entrance layer nodes, $\mathrm{w}_{\mathrm{j} s}$ : weight related to ${ }_{\text {is }}$ enter in $\mathrm{i}$-th node, ${ }_{\text {is }}$ : weight of $\mathrm{i}$-th node, $\mathrm{b}_{0}$ and $b_{\mathrm{j} 0}$ : bias of middle and output layers, respectively, $\varnothing_{2}$ and $\emptyset_{1}$ : activation functions of hidden and output layers.

$$
\hat{\mathrm{y}}_{\mathrm{i}}=\emptyset_{2}\left(b_{0}+\sum_{j=1}^{M} w_{j} \emptyset_{1}\left(b_{j 0}+\sum_{s=1}^{p} x_{i s} w_{j s}\right)\right) i=1,2, \ldots, n
$$


The activation function of hidden layer usually is non-linear (hyperbolic tangent or sigmoid), and conversion function of output layer can be linear or non-linear $^{[15,27]}$.

The aim of ANN is calculating the proper weight for network. One way of measuring the weights is backpropagation algorithm. Back-propagation rule consists of two paths. One path is forward path, in which entrance vector applies to perceptron network, and its effect expands into output layer through a middle layer. The constructed output vector in the output layer makes the real response of the perceptron network, which is named as the backward path, in which the parameters of network are considered fixed. In this path, in contrast to the going path, the parameters of perceptron network are changed and adjusted. This adjustment is done according to the error correction law. These paths are repeated until the parameter estimates (bias and weight) are adjusted. The process of measuring the proper weight is considered as the learning process. For doing this process, weight coefficients will be changed to reduce the goal function of network, which is considered as mean square error $^{[28,29]}$.

\section{Support vector machine}

SVM is a data mining tool and is a supervised classification technique. This method can be used for prediction when the outcome variable is binary. SVM constructs multi-dimensional hyper-planes separating the two classes while maximizing the margin between the two classes. SVM uses kernel functions and has the ability to discriminate between classes that are not linearly separable ${ }^{[30]}$. The dataset is divided into two sets with the training dataset comprising of 6654 cases (70\%) and testing dataset containing 2874 cases (30\%). Each model is developed using the training dataset and tested using the testing dataset ${ }^{[31]}$. In case of each model, the incidence of type 2 diabetes is predicted, and confusion matrix is constructed in order to measure the accuracy of the model. Accuracy is measured as the proportion of cases classified correctly. Sensitivity is measured by the proportion of positive cases classified correctly, while specificity is determined by the proportion of negative cases classified correctly $^{[7]}$. Mathematically, if TP stands for true positive, TN for true negative, FP for false positive, and $\mathrm{FN}$ for false negative, then accuracy = $(\mathrm{TP}+\mathrm{TN}) /(\mathrm{TP}+\mathrm{FP}+\mathrm{TN}+\mathrm{FN})$, sensitivity $=\mathrm{TP} /(\mathrm{TP}$ $+\mathrm{FN})$, and specificity $=\mathrm{TN} /(\mathrm{FP}+\mathrm{TN})$.

\section{Statistical analysis}

The data were analyzed using $\mathrm{R}$ version 3.0.2. All variables were analyzed to generate descriptive statistics, chi-square tests, independent sample $t$-tests for variables with a normally distributed variables, and Mann-Whitney tests for non-normally distributed variables. MLR was used to identify factors that are strongly associated with type 2 DM.

\section{RESULTS}

\section{Characteristics of the population}

Descriptive statistics for anthropometric and biochemical characteristics are reported in Tables 1 and 2, respectively. The number of subjects having type 2 diabetes was 1361, which were selected from 9528 subjects. The mean age of diabetic individuals was higher than non-diabetic individuals (52.01 \pm 7.2 vs. $47.70 \pm 8.1)$. Of 1361 diabetic individuals, 843 $(61.9 \%)$ were female, $1239(91 \%)$ were married, and $783(57.5 \%)$ were unemployed. Subjects having DM were significantly $(p<0.05)$ older and had higher BMI, systolic blood pressure, diastolic blood pressure, serum TC, and LDL cholesterol, as well as a lower level of HDL-C, compared to the individuals without DM (Table 1).

\section{Multiple logistic regression model}

MLR identified a strong association between diabetes and the following characteristics: age, family history of diabetes, BMI, systolic blood pressure, diastolic blood pressure, hs-CRP, TG, and PAL. (Fig. 1A) The ORs of age (95\% CI: 1.04-1.06, $p<0.001)$, family history of diabetes (95\% CI: 2.5-3.16, $p<$ 0.001 ), and hs-CRP (95\% CI: 1.01-1.02, $p<0.001$ ) were $1.05,2.81,1.02$, respectively. The results showed that for one unit of increase in hs-CRP, the chance for diabetes is 1.02 units. The results are reported in Table 2 .

\section{Results of artificial neural network model}

For designing the neural network and finding the best model, we performed the test on the least square error function. At the beginning, the learning rate and the number of neurons were considered as 0.05 and 10 , respectively. Sigmoid activation function was used in a hidden layer, and linear activation function was used in the output layer. Gradually, the learning rate was increased up to 0.5 , and the number of neurons up to 25 increased. All the combinations of different learning rates and the number of neurons were tested.

Subsequently, according to the mean square error, the best model was selected. After considering different combinations, the three-layer perceptron network with 24 nodes in entrance layer, 20 nodes in hidden layer, and 2 nodes in output layer with the rate learning of 0.2 were used, which in comparison with other combinations had the least mean square error 
Table 1. Comparison of baseline characteristics between diabetes and non-diabetes groups

\begin{tabular}{|c|c|c|c|}
\hline Variables & Diabetes $(n=1361)$ & Non-diabetes $(n=8167)$ & $p$ value \\
\hline Age (year) & $52.01 \pm 7.2$ & $47.70 \pm 8.1$ & $<0.001$ \\
\hline BMI $\left(\mathrm{kg} / \mathrm{m}^{2}\right)$ & $28.78 \pm 4.4$ & $27.76 \pm 4.7$ & $<0.001$ \\
\hline \multicolumn{4}{|l|}{ Gender } \\
\hline Male & $518(38.1 \%)$ & $3277(40.1 \%)$ & \multirow{2}{*}{$=0.04$} \\
\hline Female & $843(61.9 \%)$ & $4890(59.9 \%)$ & \\
\hline \multicolumn{4}{|l|}{ Marriage status } \\
\hline Single & $5(0.4 \%)$ & $54(0.7 \%)$ & \multirow{4}{*}{$<0.001$} \\
\hline Married & $1239(91 \%)$ & $7636(93.5 \%)$ & \\
\hline Divorced & $21(1.5 \%)$ & $111(1.4 \%)$ & \\
\hline Widow & $96(7.1 \%)$ & $366(4.5 \%)$ & \\
\hline \multicolumn{4}{|l|}{ Education Level } \\
\hline Low & $878(64.5 \%)$ & $4319(52.9 \%)$ & \multirow{3}{*}{$<0.001$} \\
\hline Moderate & $374(27.5 \%)$ & $2912(35.7 \%)$ & \\
\hline High & $109(8.0 \%)$ & $936(11.5 \%)$ & \\
\hline \multicolumn{4}{|l|}{ Occupation status } \\
\hline Employment & $400(29.4 \%)$ & $3125(38.3 \%)$ & \multirow{3}{*}{$<0.001$} \\
\hline Unemployment & $783(57.5 \%)$ & $4283(52.4 \%)$ & \\
\hline Retired & $178(13.1 \%)$ & $759(9.3 \%)$ & \\
\hline \multicolumn{4}{|l|}{ Smoking status } \\
\hline Yes & $272(20.0 \%)$ & $1775(21.7 \%)$ & \multirow{2}{*}{$=0.05$} \\
\hline No & $1089(80.0 \%)$ & $6392(78.3 \%)$ & \\
\hline \multicolumn{4}{|c|}{ Family history of diabetes } \\
\hline Yes & $647(47.5 \%)$ & $1994(24.4 \%)$ & \multirow{2}{*}{$<0.001$} \\
\hline No & $714(52.5 \%)$ & $6173(75.6 \%)$ & \\
\hline \multicolumn{4}{|l|}{ Depression } \\
\hline Yes & $461(33.9 \%)$ & $2226(27.3 \%)$ & \multirow{2}{*}{$=0.001$} \\
\hline No & $900(66.1 \%)$ & $5941(72.7 \%)$ & \\
\hline $\mathrm{SBP}(\mathrm{mmHg})$ & $128.81 \pm 18.4$ & $121.14 \pm 18.2$ & $<0.001$ \\
\hline DBP (mmHg) & $81.36 \pm 10.4$ & $78.91 \pm 11.1$ & $<0.001$ \\
\hline Cholesterol (mg/dL) & $201.46 \pm 46.3$ & $189.69 \pm 37.8$ & $<0.001$ \\
\hline LDL (mg/dL) & $120.49 \pm 39.1$ & $116.74 \pm 34.6$ & $<0.001$ \\
\hline HDL (mg/dL) & $42.81 \pm 9.6$ & $42.73 \pm 9.9$ & $=0.004$ \\
\hline PAL (h per week) & $1.60 \pm 0.64$ & $1.59 \pm 0.86$ & $=0.04$ \\
\hline hs-CRP (mg/l) & $2.7[1.3-4.9]^{*}$ & $1.56[0.95-3.23]^{*}$ & $<0.001$ \\
\hline TG (mg/dL) & $160[105-222]^{*}$ & $117[82-165]^{*}$ & $<0.001$ \\
\hline
\end{tabular}

*Median (IQR) 
Table 2. Multiple logistic regression analysis on the influential factors of type 2 diabetes in training dataset

\begin{tabular}{|c|c|c|c|}
\hline Variables & B (SE) & OR $(95 \% \mathrm{CI})$ & $P$ value \\
\hline Age(year) & $0.05(0.004)$ & $1.05(1.04-1.06)$ & $<0.001$ \\
\hline \multicolumn{4}{|l|}{ Education } \\
\hline High & & Reference & $=0.27$ \\
\hline Moderate & $0.12(0.11)$ & $1.13(0.91-1.39)$ & $=0.59$ \\
\hline Low & $-0.04(0.07)$ & $0.96(0.84-1.11)$ & \\
\hline \multicolumn{4}{|l|}{ Occupation } \\
\hline Unemployment & & Reference & \\
\hline Retired & $0.16(0.11)$ & $1.18(0.94-1.47)$ & $=0.15$ \\
\hline Employment & $0.46(0.12)$ & $1.59(1.27-1.99)$ & $<0.001$ \\
\hline \multicolumn{4}{|l|}{ Married status } \\
\hline Divorced & & Reference & \\
\hline Married & $-0.22(0.24)$ & $0.80(0.50-1.28)$ & $=0.36$ \\
\hline Single & $-0.67(0.54)$ & $0.51(0.17-1.48)$ & $=0.21$ \\
\hline Widow & $-0.23(0.26)$ & $0.79(0.47-1.33)$ & $=0.37$ \\
\hline \multicolumn{4}{|l|}{ Smoking status } \\
\hline No & & Reference & \\
\hline Yes & $-0.11(0.07)$ & $0.89(0.77-1.03)$ & $=0.13$ \\
\hline \multicolumn{4}{|c|}{ Family history of diabetes } \\
\hline No & & Reference & \\
\hline Yes & $1.03(0.06)$ & $2.81(2.5-3.16)$ & $<0.001$ \\
\hline \multicolumn{4}{|l|}{ Depression } \\
\hline No & & Reference & \\
\hline Yes & & $0.76(0.61-0.95)$ & $<0.001$ \\
\hline BMI $\left(\mathrm{kg} / \mathrm{m}^{2}\right)$ & $0.02(0.007)$ & $1.02(1.01-1.02)$ & $<0.001$ \\
\hline $\mathrm{SBP}(\mathrm{mmHg})$ & $0.01(0.002)$ & $1.02(1.01-1.02)$ & $<0.001$ \\
\hline DBP (mmHg) & $-0.01(0.004)$ & $0.99(0.98-0.99)$ & $=0.009$ \\
\hline LDL (mg/dL) & $-0.003(0.002)$ & $0.99(0.99-1.001)$ & $=0.1$ \\
\hline HDL (mg/dL) & $-0.003(0.004)$ & $0.99(0.99-1.005)$ & $=0.47$ \\
\hline Cholesterol (mg/dL) & $0.005(0.002)$ & $1.005(1.001-1.008)$ & $=0.007$ \\
\hline hs-CRP (mg/l) & $0.02(0.003)$ & $1.02(1.01-1.02)$ & $<0.001$ \\
\hline $\mathrm{TG}(\mathrm{mg} / \mathrm{dL})$ & $0.003(0.0001)$ & $1.003(1.002-1.004)$ & $<0.001$ \\
\hline PAL (h per week) & $0.001(0.0001)$ & $1.001(1.001-1.003)$ & $=0.001$ \\
\hline
\end{tabular}

SE, standard error

Table 3. The mean predicted error according to the number of hidden layer nodes by neural network

\begin{tabular}{|c|c|c|c|c|c|c|c|}
\hline & \multicolumn{6}{|c|}{ Number of hidden layer nodes } & \multirow{2}{*}{ Learning rate } \\
\hline & 20 & 18 & 16 & 14 & 12 & 10 & \\
\hline \multirow{4}{*}{ 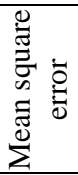 } & 27.04 & 27.12 & 27.52 & 27.56 & 27.74 & 28.51 & 0.05 \\
\hline & 26.84 & 26.94 & 27.56 & 27.44 & 27.80 & 28.46 & 0.01 \\
\hline & 26.8 & 27.23 & 27.39 & 27.54 & 27.87 & 28.38 & 0.20 \\
\hline & 26.94 & 27.37 & 27.38 & 27.51 & 27.86 & 28.37 & 0.50 \\
\hline
\end{tabular}



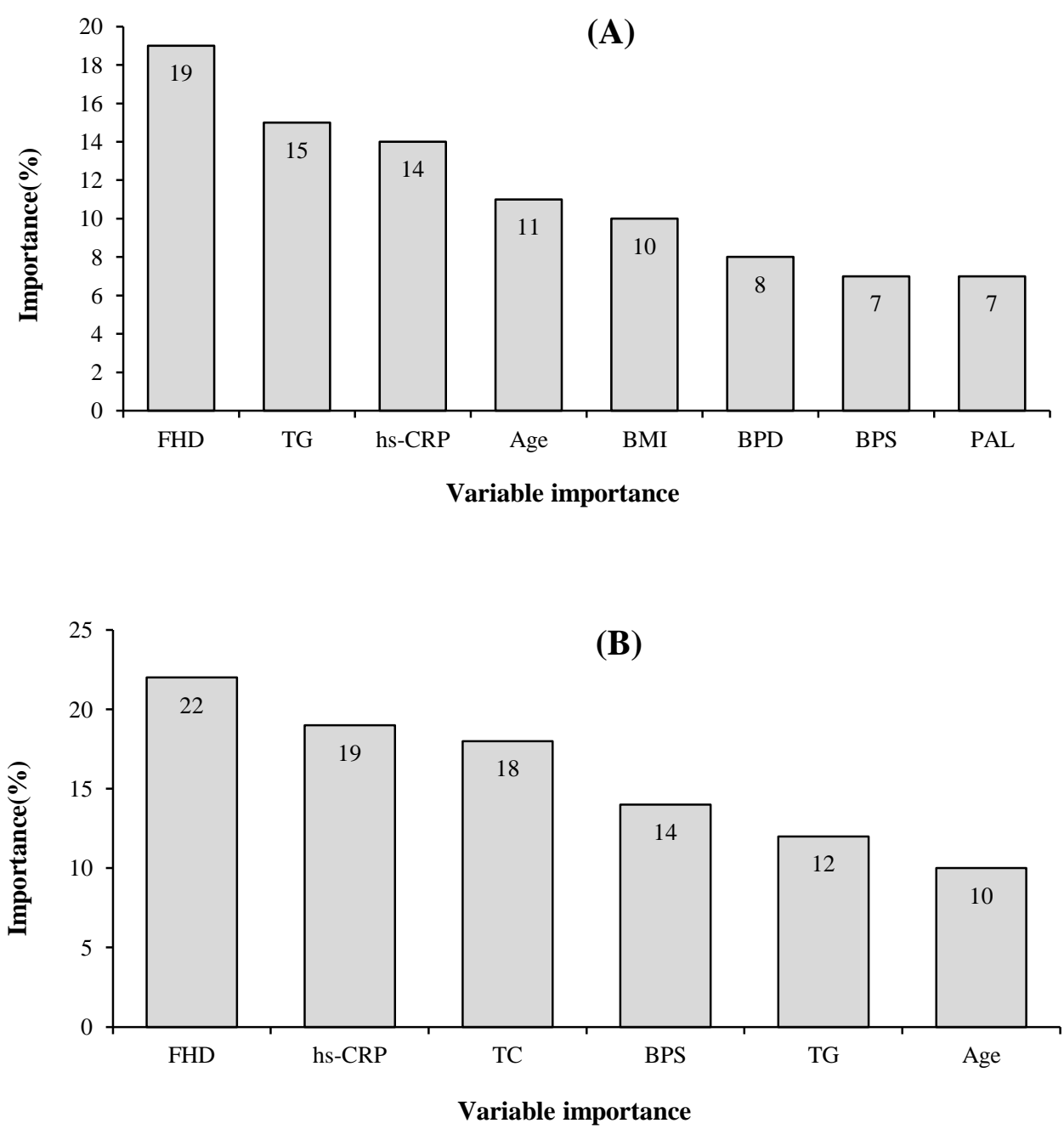

(C)

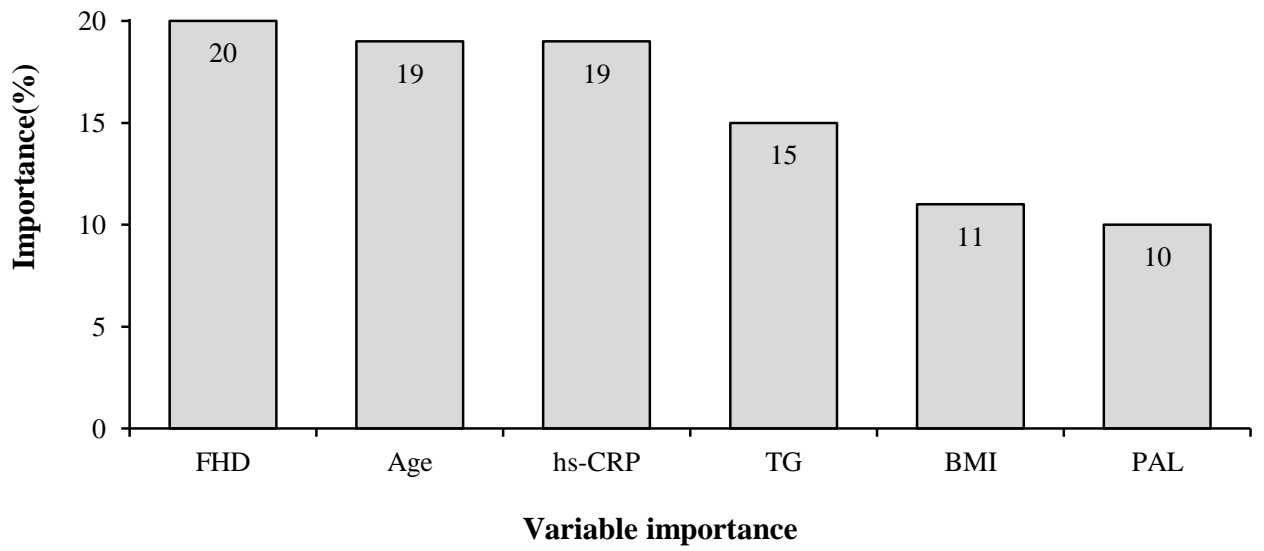

Fig. 1. The importance of input variables in MLR (A), ANN (B), and SVM (C) models. 


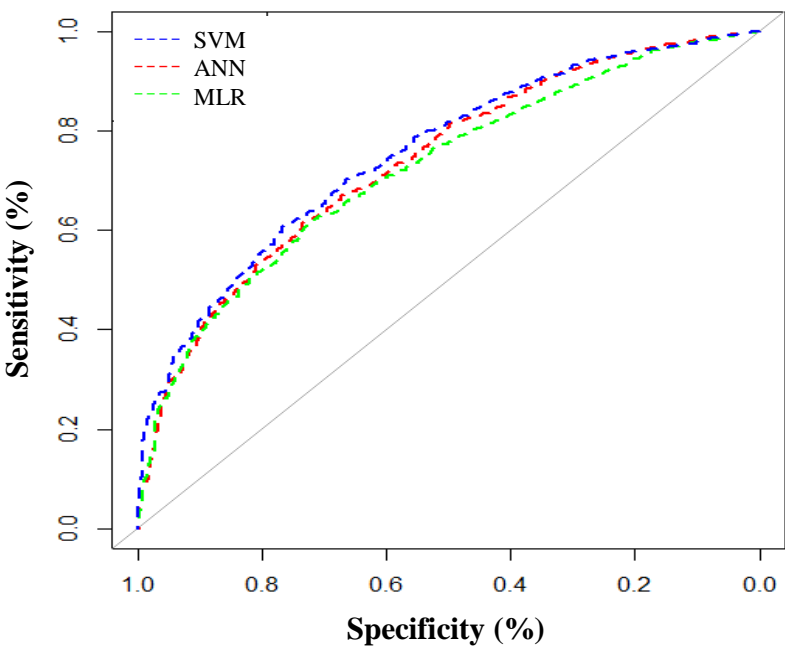

Fig. 2. ROC curves of the SVM, ANN, and MLR models in testing dataset.

(Table 3). The order of important variables in the ANN model containing six parameters was as follow: family history of diabetes, hs-CRP, TC, BPS, TG, and age risk factor diabetic (Fig. 1B).

\section{Results of support vector machines model}

SVM applied the same 17 characteristics used by ANN and tried sigmoid as well as polynomial kernel functions to identify the best kernel function. Based on the results of the SVM model, family history of diabetes, age, hs-CRP, TG, BMI, and PAL were the most important risk factors related to type 2 diabetes (Fig. 1C). Table 4 summarizes the results on accuracy, sensitivity, specificity, and area under the ROC curve for these three models. As shown in the Table, SVM model had the best performance. ROC curve for the three models is displayed in Figure 2.

\section{DISCUSSION}

In the present study, we developed and explored the effectiveness of three models of data mining, including ANN, MLR, and SVM, in order to identify some potential factors associated with type 2 diabetes in a large population consisted of 9582 subjects with and without diabetes. In line with the previous studies, our results indicated that ANN is better than MLR and SVM for identifying associated risk factors of type 2 diabetes $^{[10,19]}$

MLR, ANN, and SVM were applied on training data set, and all models were used for the evaluation of testing data set. Based on demographic and biochemical markers, our findings showed that the ANN model had a higher predictive accuracy in comparison to MLR and SVM models.

The sensitivity and specificity are two important factors for the validity of a model ${ }^{[32]}$; therefore, these two criteria were calculated for the three models. The results revealed that $A N N$ model had more sensitivity and specificity than MLR model and less sensitivity than SVM, which are in agreement with the findings of Meng et $a l^{[8]}$ and Sedehi et $a l^{[29]}$ studies. Investigations have also suggested that ANN could give a better prediction value than $\mathrm{MLR}^{[8,17]}$. Those studies have compared ANN and MLR models using demographic and anthropometric variables, but we have used biochemical parameters. Moreover, we found that in the ANN model, the family history of diabetes and hs-CRP had a more important role in the identification of individuals with type 2 diabetes, while in MLR model, family history of diabetes, TG and hsCRP and in SVM model, the family history of diabetes, age, and hs-CRP play a key role in determining diabetes risk, showing that hs-CRP is an important and a common factor in three models for determining the individuals with type 2 diabetes A major strength of the present study is that it was performed in a large number of samples, thus providing a new insight to the application of ANN, MLR, and SVM for investigating new potential risk factors associated with type 2 diabetes in a representative sample of the Iranian population. However, future studies based on cohort studies are needed to give better estimates of the accuracy, sensitivity, specificity, and area under the ROC curve for ANN.

Table 4. The performance of three models for identifying associated risk factors of type 2 diabetes

\begin{tabular}{llll}
\hline Model (\%) & ANN (95\% CI) & MLR (95\% CI) & SVM (95\% CI) \\
\hline Sensitivity & $63.1(59.8-67.5)$ & $60.1(58.4-63.1)$ & $64.5(59.8-66.4)$ \\
Specificity & $81.2(78.4-84.6)$ & $80.5(76.4-85.3)$ & $78.9(76.4-81.7)$ \\
Accuracy & $78.7(73.5-82.6)$ & $77.7(73.5-80.9)$ & $76.8(73.5-80.9)$ \\
AUC & $71.5(68.0-75.9)$ & $70.4(68.6-73.9)$ & $73.1(69.2-77.6)$
\end{tabular}

AUC, the area under the ROC curve 
Although we determined and identified the risk factors associated with type 2 diabetes through ANN, SVM, and MLR models using demographic, anthropometric, and biochemical factors of a large population, more investigations are required to assess the clinical applicability of these three models for evaluation of new risk factors of type 2 diabetes.

\section{ACKNOWLEDGMENTS}

This study was financially supported by the Biochemistry of Nutrition Research Center of Mashhad University of Medical Sciences, Mashahd, Iran.

CONFLICT OF INTEREST. None declared.

\section{REFERENCES}

1. Guariguata L, Whiting DR, Hambleton I, Beagley J, Linnenkamp U, Shaw J. Global estimates of diabetes prevalence for 2013 and projections for 2035. Diabetes research and clinical practice 2014; 103(2): 137-149.

2. Hemmati M, Zohoori E, Mehrpour O, Karamian M, Asghari S, Zarban A, Nasouti R. Anti-atherogenic potential of jujube, saffron and barberry: anti-diabetic and antioxidant actions. EXCLI journal 2015; 14: 908-915.

3. Abbasi A, Peelen LM, Corpeleijn E, van der Schouw YT, Stolk RP, Spijkerman AM, van der ADL, Moons KG, Navis G, Bakker SJ, Beulens JW. Prediction models for risk of developing type 2 diabetes: systematic literature search and independent external validation study. $B M J$ 2012; 345: e5900.

4. Organization WH. Screening for type 2 diabetes: report of a World Health Organization and International Diabetes Federation meeting. Reterieved from: http://apps.who.int/iris/handle/10665/68614

5. Schulze MB, Heidemann C, Schienkiewitz A, Bergmann MM, Hoffmann K, Boeing H. Comparison of anthropometric characteristics in predicting the incidence of type 2 diabetes in the EPIC-Potsdam study. Diabetes care 2006; 29(8): 1921-1923.

6. Lönn M, Mehlig K, Bengtsson C, Lissner L. Adipocyte size predicts incidence of type 2 diabetes in women. The FASEB journal 2010; 24(1): 326-331.

7. Bellazzi R, Zupan B. Predictive data mining in clinical medicine: current issues and guidelines. International journal of medical informatics 2008; 77(2): 81-97.

8. Meng XH, Huang YX, Rao DP, Zhang Q, Liu Q. Comparison of three data mining models for predicting diabetes or prediabetes by risk factors. The Kaohsiung journal of medical sciences 2013; 29(2): 93-99.

9. Chen HY, Chuang CH, Yang YJ, Wu TP. Exploring the risk factors of preterm birth using data mining. Expert systems with applications 2011; 38(5): 5384-5387.

10. Tayefi M, Tajfard M, Saffar S, Hanachi P,
Amirabadizadeh AR, Esmaeily H, Taghipour A, Ferns GA, Moohebati M, Ghayour-Mobarhan M. hs-CRP is strongly associated with coronary heart disease (CHD): A data mining approach using decision tree algorithm. Computer methods and programs in biomedicine 2017; 141: 105-109.

11. Agresti A. An Introduction to Categorical Data Analysis. New York: Wiley; 1996.

12. Amirabadizadeh A, Nezami H, Vaughn MG, Nakhaee $\mathrm{S}$, Mehrpour O. Identifying risk factors for drug use in an Iranian treatment sample: A prediction approach using decision trees. Substance use and misuse 2017: 111.

13. Karimi-Alavijeh F, Jalili S, Sadeghi M. Predicting metabolic syndrome using decision tree and support vector machine methods. ARYA atherosclerosis 2016; 12(3): 146-152.

14. Saha S, Bhattacharya S, Konar A. A Novel Approach to Gesture Recognition in Sign Language Applications Using AVL Tree and SVM. Progress in Intelligent Computing Techniques: Theory, Practice, and Applications. Singapore: Springer; 2018.

15. Lee TT, Liu CY, Kuo YH, Mills ME, Fong JG, Hung C. Application of data mining to the identification of critical factors in patient falls using a web-based reporting system. International journal of medical informatics 2011; 80(2): 141-150.

16. Delen D, Walker G, Kadam A. Predicting breast cancer survivability: a comparison of three data mining methods. Artificial intelligence in medicine 2005; 34(2): 113-127.

17. Wang C, Li L, Wang L, Ping Z, Flory MT, Wang G, Xi $\mathrm{Y}, \mathrm{Li} \mathrm{W}$. Evaluating the risk of type 2 diabetes mellitus using artificial neural network: an effective classification approach. Diabetes research and clinical practice 2013; 100(1): 111-118.

18. Bassuk SS, Manson JE. Epidemiological evidence for the role of physical activity in reducing risk of type 2 diabetes and cardiovascular disease. Journal of applied physiology (1985) 2005; 99(3): 1193-1204.

19. Salmerón J, Hu FB, Manson JE, Stampfer MJ, Colditz GA, Rimm EB, Willett WC. Dietary fat intake and risk of type 2 diabetes in women. The American journal of clinical nutrition 2001; 73(6): 1019-1026.

20. Aslan K, Bozdemir H, Sahin C, Ogulata SN. Can neural network able to estimate the prognosis of epilepsy patients accorrding to risk factors? Journal of medical systems 2010; 34(4): 541-550.

21. Tayefi M, Saberi-Karimian M, Esmaeili $H$, Amirabadizadeh A, Ebrahimi M, Mohebati M, HeidariBakavoli A, Azarpajouh MR, Heshmati M, Safarian M, Nematy M, Parizadeh SMR, Ferns GA, GhayourMobarhan M. Evaluating of associated risk factors of metabolic syndrome by using decision tree. Comparative clinical pathology 2018; 27(1): 215-223.

22. Su CT, Yang CH, Hsu KH, Chiu WK. Data mining for the diagnosis of type II diabetes from three-dimensional body surface anthropometrical scanning data. Computers \& mathematics with applications 2006; 51(6): 1075-1092. 
23. Tayefi M, Esmaeili H, Saberi Karimian M, Amirabadi Zadeh A, Ebrahimi M, Safarian M, Nematy M, Parizadeh SMR, Ferns GA, Ghayour-Mobarhan M. The application of a decision tree to establish the parameters associated with hypertension. Computer methods and programs in biomedicine 2017; 139: 83-91.

24. Ghayour-Mobarhan M, Moohebati M, Esmaily H, Ebrahimi M, Parizadeh SM, Heidari-Bakavoli AR, Safarian M, Mokhber N, Nematy M, Saber H, Mohammadi M, Andalibi MS, Ferns GA, Azarpazhooh MR. Mashhad stroke and heart atherosclerotic disorder (MASHAD) study: design, baseline characteristics and 10 -year cardiovascular risk estimation. International journal of public health 2015; 60(5): 561-572.

25. Chou SM, Lee TS, Shao YE, Chen IF. Mining the breast cancer pattern using artificial neural networks and multivariate adaptive regression splines. Expert systems with applications 2004; 27(1): 133-142.

26. Haykin S. Neural networks and learning machines. USA: Prentice Hall; 2009.

27. Hagan MT, Demuth HB, Beale MH, De Jess O. Neural Network Design. USA: Martin Hagan; 2014.

28. Adavi M, Salehi M, Roudbari M. Artificial neural networks versus bivariate logistic regression in prediction diagnosis of patients with hypertension and diabetes. Medical journal of the Islamic Republic of Iran 2016; 30: 312.

29. Sedehi M, Mehrabi Y, Kazemnejad A, Joharimajd V, Hadaegh F. Artificial neural network design for modeling of mixed bivariate outcomes in medical research data. Iranian journal of epidemiology 2011; 6(4): 28-39.

30. Yu W, Liu T, Valdez R, Gwinn M, Khoury MJ. Application of support vector machine modeling for prediction of common diseases: the case of diabetes and pre-diabetes. BMC medical informatics and decision making 2010; 10:16.

31. Ramezankhani A, Pournik O, Shahrabi J, Azizi F, Hadaegh F, Khalili D. The impact of oversampling with SMOTE on the performance of 3 classifiers in prediction of type 2 diabetes. Medical decision making 2016; 36(1): 137-144.

32. Ho WH, Lee KT, Chen HY, Ho TW, Chiu HC. Diseasefree survival after hepatic resection in hepatocellular carcinoma patients: a prediction approach using artificial neural network. PLoS one 2012; 7(1): e29176. 